\title{
Dielectric and Mechanical Properties of Local Commercial Single Walled Carbon Nanotubes for Medical Applications: A Practical Approach
}

\author{
Moustafa Hussein Moustafa \\ Biomedical Physics Department, Alexandria University, Alexandria, Egypt
}

Email address:

moustafa-hm@alexu.edu.eg

\section{To cite this article:}

Moustafa Hussein Moustafa. Dielectric and Mechanical Properties of Local Commercial Single Walled Carbon Nanotubes for Medical Applications: A Practical Approach. American Journal of Nanosciences. Vol. 7, No. 1, 2021, pp. 1-5. doi: 10.11648/j.ajn.20210701.11

Received: December 10, 2020; Accepted: December 17, 2020; Published: January 22, 2021

\begin{abstract}
Owing to their exceptional electrical and mechanical properties, low weights and availability of their synthesis and purification carbon nanotubes (CNTs) became the focus of scientists in many medical and electronic applications, and reinforcement of various materials. A close understanding of structure-properties of (CNTs) will be necessary for the development of carbon-nanotube applications. High stiffness and tensile strength of CNTs provide mechanical stability for electric nano-circuits formed by CNTs. Meanwhile, at earlier time of their application, researchers main concern was dedicated for preparation methods of CNTs. Eventually the structural and physical characteristics of CNTs became more attractive for scientist proper applications. Herein the current work introduce a practical approach for screening the dielectric and mechanical properties of local commercial purchased single walled carbon nanotubes for medical applications. Dielectric properties of CNTS were expressed in term of electrical relative permittivity, and electrical conductivity. The dielectric relaxation obeys ordinary alpha and beta relaxation of dielectrics at current frequency range. Besides the mechanical properties in term of dimetral tensile and compression strength that are expressed by Young's modulus that is closer to graphite. As a results, we highly recommend these commercial (SWCNTs) from our local suppliers for further medical applications.
\end{abstract}

Keywords: Carbon Nanotubes, Tensile Strength, Dielectrics, Graphite, Permittivity

\section{Introduction}

The plethora of structural, mechanical, and electronic properties for tiny rolled graphite sheet, carbon nanotubes (CNTs), have been nominated them to be the premier additives to various structural materials in electronics, optics, plastics, and other nanotechnology materials. In early nineteenths (CNTs) were fabricated by the Japanese scienticst Ijima [1]. Researchers mainly were concerned with the fabrication methods, as it was a challenge to yield (CNTs) with desired dimensions and degree of purity. Chirality structure and diameter of carbon sheet are the crucial for CNTs properties $[2,3]$.

By the beginning of 21st century, CNTs were incorporated in many medical pharmaceutical industries especially in drug delivery system and become the focus of attention by scientists in a variety of disciplines. CNTs were applied first in cancer and infection through binding to antineoplastic and antibiotic drugs. Consequently, CNTs were linked to biomolecules such as: genes, proteins, DNA, antibodies, vaccines, biosensors, cells. Moreover, they have shown as Excellency in enantiomer separation of chiral drugs and chemicals in pharmaceutical industry and drug extraction as well $[4,5]$.

Thanks to their unique structure of high surface area, chemical stability, and rich electronic polyaromatic structure, CNTs were became excellent vehicles for drug delivery by direct cell penetrating with keeping the drug intact without metabolism during transport in the body. These criteria of CNT have paved the way for new drug preparations protocols that is radically changed concepts of pharmacology $[6,7]$.

Eventually, new dielectric properties have made it possible to design CNTs composites. That is why a number of researchers have changed the lane towards novel properties other than fabrication issues. Dielectric properties were the 
main goal of studies that made CNTs a semiconductor device. Electrical permittivity and conductivity over a great scale of frequency were studies. Hereby, a practical approach for screening the dielectric and mechanical properties of commercial single walled carbon nanotubes (SWCNTs) for medical applications purchased from local supplier $[8,9,11]$.

\section{Materials and Methods}

\subsection{Carbon Nanotubes (CNTS)}

Carbon nanotubes (CNTs) were purchased from nanotech ${ }^{\circledR}$ (Giza, Egypt) with a high-yield catalytic process based on chemical vapor deposition with a purity of carbon content more than $95 \mathrm{wt} \%$. CNTs analysis tests were carried out at Institute of Advanced Technology Research and New Materials, City of Scientific research and Technological applications, Borg Al Arab, Alexandria Governorate.

\subsection{Electron Microscopy}

The size and surface morphology of CNTs samples were monitored using transmission (TEM model: JEOL 1400 Plus, Japan) and scanning (SEM model: JSM-5300, JEOL, Japan). Measurements were processed by drop coating method on carbon coated copper grids kept under vacuum. The average diameters of CNTs were deduced on the captured microscopic photographs, as seen in Figure 1.

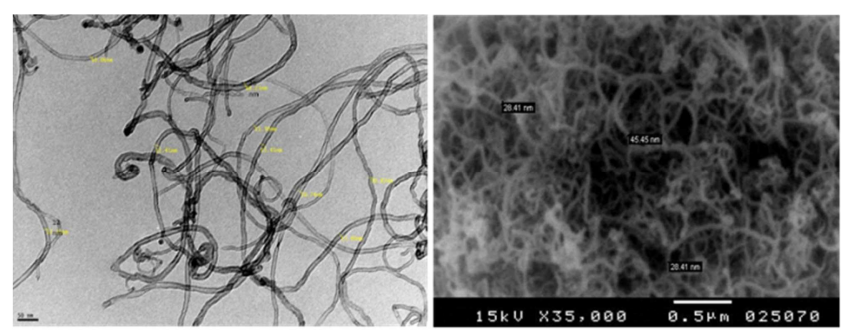

Figure 1. Photographs of TEM (A), and SEM (B) for CNTs powder.

\subsection{Fourier Transform Infrared Analysis (FT-IR)}

(CNTs) active functional groups were analyzed by using FT-IR (model: 8400 s, Shimadzu corporation, Japan). The samples were mixed with Potassium bromide $(\mathrm{KBr})$ powder then placed in a sample cup. The diffused reflected spectra converted into transmission spectra to be compared with a maximum resolution of $0.85 \mathrm{~cm}-1$ within the range of 400 $4000 \mathrm{~cm}-1$. The ratios of band integrated intensities and areas of corresponding structure were calculated.

\subsection{X-ray Diffraction (XRD)}

Here in crystalline size was measured using XRD-6100 X-ray Diffractometer (SHIMADZU Corporation., Japan) to check the analysis of impurities for CNTs. The changes in the diffracted X-ray intensities are measured at different angles and plotted against the rotation angles of the sample. The peaks positions provide qualitative analysis, lattice constant determination and/or stress determination of the sample.

\subsection{Dielectric Measurements}

Dielectric measurements were made in frequency range 42 $\mathrm{KHz}$ to $5 \mathrm{MHz}$ using LCR meter (Hioki, 3532, LCR Hitester, Japan). The (CNTs) sample of was placed in a homemade measuring cell was designed as two cubic silver electrodes of area (A) $1 \times 1 \mathrm{~mm}^{2}$ and $5 \mathrm{~mm}$ apart (d). The capacitance (C) and resistance $(\mathrm{R})$ was recorded over the frequency range at a constant temperature of $24 \pm 0.1^{\circ} \mathrm{C}$. Dielectric constant $(\varepsilon)$ (relative permittivity), was calculated room the equations (1). Electrical conductivity $(\sigma)$ was also calculated from Equation 2 as function of frequency of the sample was measured at each frequency and the each run was repeated three times (8).

$$
\begin{aligned}
& \varepsilon=c \frac{d}{A} \\
& \sigma=\frac{1}{R} \cdot \frac{d}{A}
\end{aligned}
$$

Pellets formation ${ }^{(13)}$

Pellet formation was adopted from buckpaper (buckytube) formation. buckytube is a frit-compression system was adapted from a Solid phase extraction column as alternative casting method was developed in 2008. Hydrated carbon nanotubes are squeezed between two polypropylene frits (70 micrometre pore diameter) on flat bottom and a syringe rubber as in Figure 2. The formed disk was dry overnight to evaporate water content leaving carbon nanotubes to be pressed together.

This method produces buckypaper that did not require the use of surfactants (like Triton X) or the acid oxidation of carbon nanotubes and avoid toxic effect on biological cells. All pellets will be well shaped, polished and cleaned its surface using ultrasound cleaner.

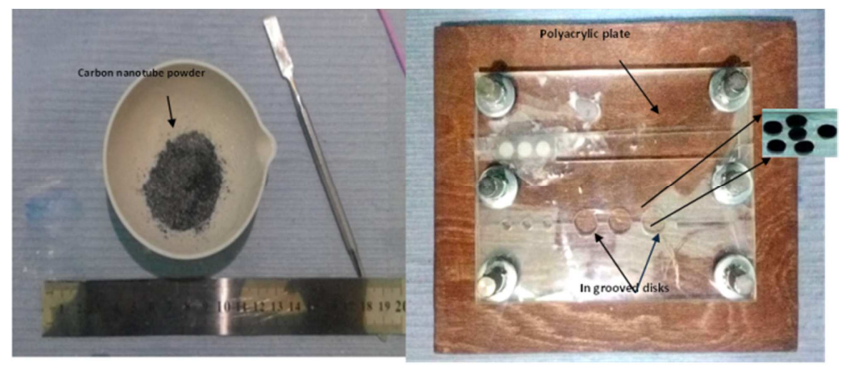

Figure 2. Concave carbon nanotubes buchydisc squeezed between syringe polypropylene and polyacrylic groove.

\subsection{Compressive Strength}

Compressive strength is the capability of CNTs pellet to withstand the original sizes against compressing loads. CS was carried out by universal test machine (model: 5KN AG-IS, SHIMADZU, Japan). The specimen was loaded between pistons at the same rate with gradual loads with crosshead speed rate of $0.75 \mathrm{~mm} / \mathrm{min}$ until fracture as in Figure 3.

\subsection{Diametral Tensile Strength}

Diametral Tensile strength test is to investigate the longitudinal elongation of brittle composite materials under longitudinal compressive loading. This test was performed by 
universal testing machine (model: 5KN AG-IS, SHIMADZU, Japan). Specimen was placed between two flat platens and increasing the load gradually until the specimen splitting.

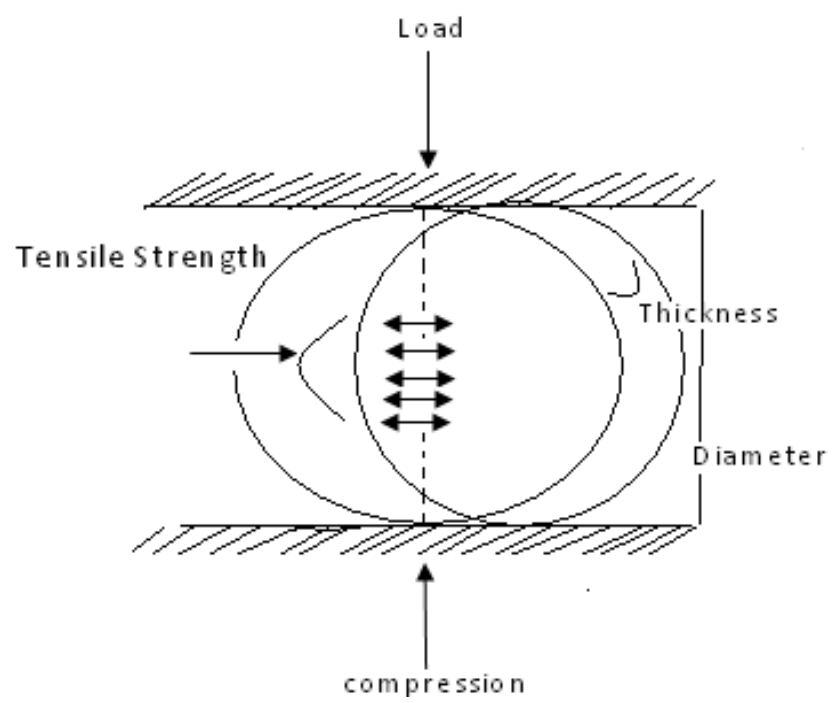

Figure 3. Diagram showing the basic idea of measuring compression strength by universal testing machine.

The modulus of elasticity (Young`s Modulus) of the composites was defined as the slope from their stress-strain curve in the elastic deformation region. While the diametral tensile strength was calculated from the following equation;

$$
D T S=\frac{2 P}{\pi d t}
$$

Where; (DTS) diametral tensile strength in (N/mm2), (P); maximu load at the fracture point in $\mathrm{N},(\mathrm{d})$, diameter of the tablet specimen in $\mathrm{mm}^{2}$, and $(\mathrm{t})$ : is the thickness of the tablet specimen in $\mathrm{mm}$.

\section{Statistical Analysis}

All descriptive parameters are expressed in mean and standard deviation. All variables were tested for normality using the Kolmogorov-Smirnov test. Consequently Parametric and non-parametric tests of variables with normal distribution were carried out.

\section{Results}

\subsection{Fourier Transform Infrared Spectroscopy (FTIR)}

CNTs absorbace spectrum is shown in Figure 4, absorbances peaks at 1631.83 and $1089.82 \mathrm{~cm}^{-1}$ are corresponding for stretching of carboxylic acids $(\mathrm{C}=\mathrm{O}$ and $\mathrm{C}-\mathrm{O})$. The peaks at 624.96 and $3794.11 \mathrm{~cm}-1$ are corresponding to the bending vibration and stretching vibration of $-\mathrm{OH}$, respectively.

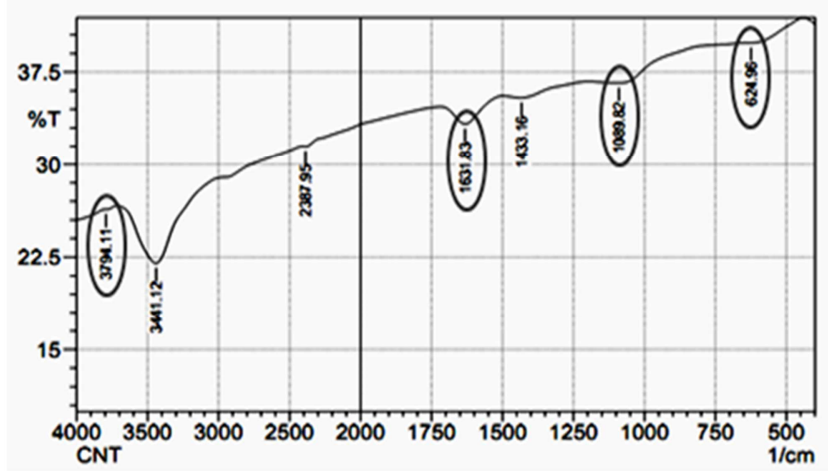

Figure 4. FTIR printout spectrum for CNTs spectrum showing absorbance peaks.

A single XRD peaks present in at scanning range of $23.94-$ $26.74(2 \theta)$ corresponds to the presence of Carbon Figure 5.

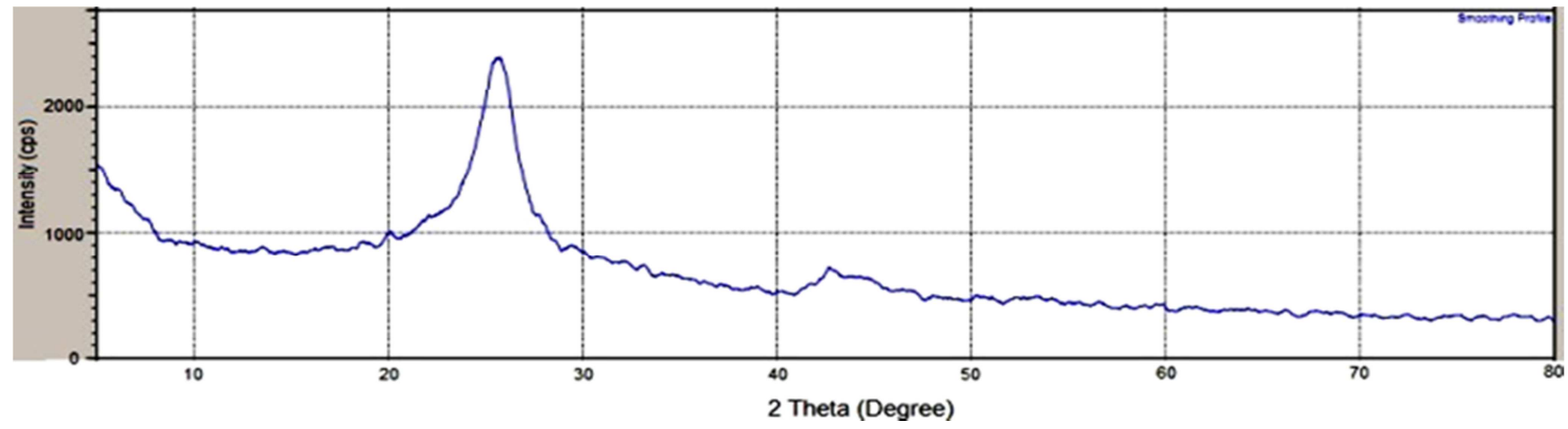

Figure 5. XRD printout for CNTs spectrum showing corresponding angle peaks.

\subsection{Dielectric Scanning}

The electrical relative permittivity $(\dot{\varepsilon})$ and electrical conductivity $(\sigma)$ are presented in. As we notice the behaviour is a regular dielectric relaxation of alpha and beta relaxation within the working frequencies at room temperature. Concerning the conductivity, there is a n opposite manner as shown Figure 6.

\subsection{Mechanical Strength}

Mechanical responses of CNTs were monitored through the diametrial tensile strength and compressive strength. In Table 1 the mean value Young's modulus was calculated from mean max stress and strain of the material. 


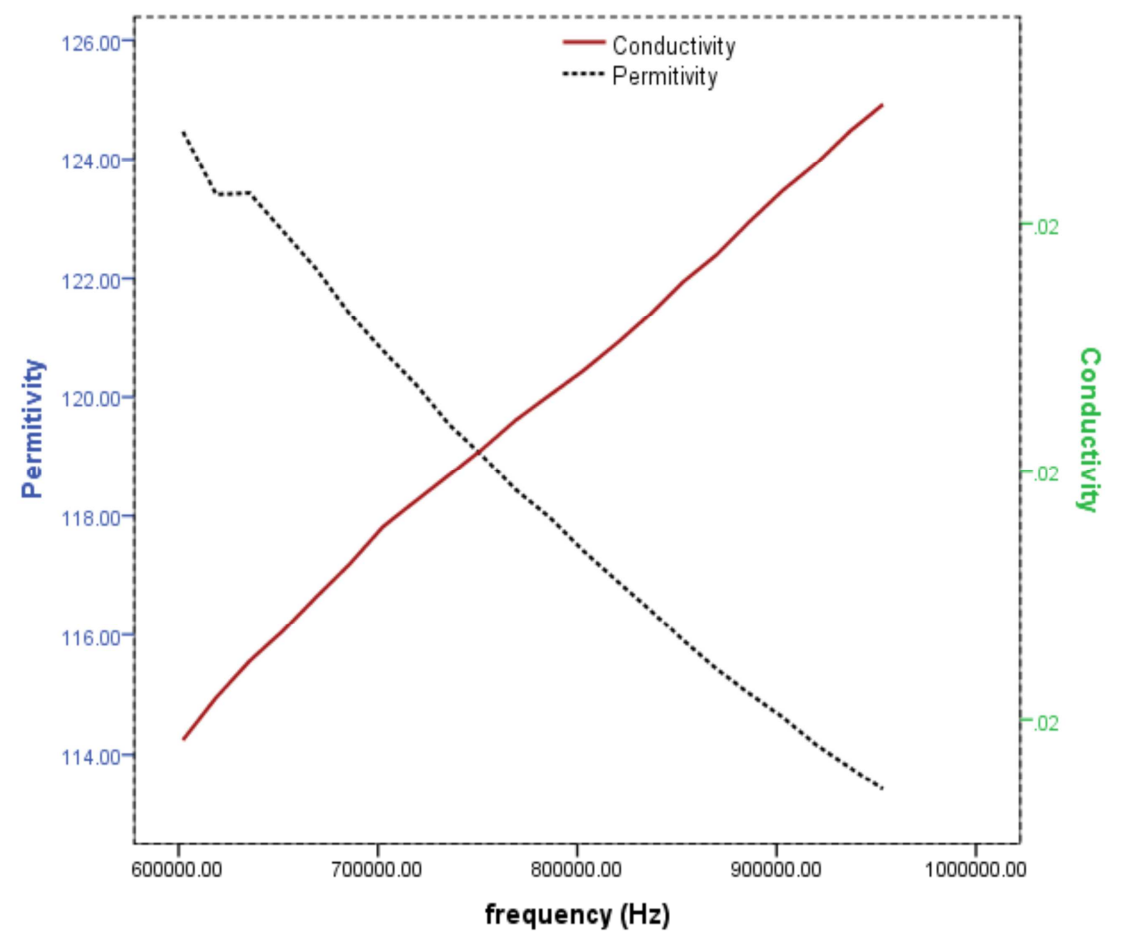

Figure 6. Dielectric spectrum with corresponding frequency-dependant relative permittivity scale (left), and electrical conductivity scale (right).

\section{Discussion}

The exceptional structural, mechanical, and electronic properties of (CNTs) have made them the premier additives to various structural materials in electronics, optics, plastics, and other nanotechnology materials. Materials strength cannot Young's modulus alone, but also related to the types, history of them. Other physical parameters may play a role such atmosphere, pressure, and the measuring system $[10,12,13]$.

Our results indicated a regular dielectric dispersion for alpha or beta relaxation. A rapid decrease in the dielectric constant may be due to the tendency materials dipole to line with the direction of the applied field in the low-frequency range. However, in higher frequency range the dipoles are hardly to orient with the applied field direction. These finding were in line with earlier works $[14,15]$.

On the other hand, electrical conductivity behaves the opposite manner. In Accordance with our results, CNTs may be transformed from the insulators to conductors according to external applied frequency $[8,16]$.

Concerning the mechanical issue, our experiments showed that Young's modulus of the current commercial grade are relatively higher as carbon and graphite as shown in Table 1.
Previous works have supported that multiwall CNTS Young's moduli are dependent upon the degree of order within the tube walls [15]. So far SWCNTs seems to behaves like flexible carbon fibers that may be applied in many medical issues $[16,17]$.

\section{Conclusion}

In summary, from our results we gather in this work that, commercial SWCNT has regular dielectric relaxation behavior in form of electrical permittivity. Besides the exceptional mechanical properties as dimetral tensile and compressive strength that are expressed by relative greater Young's modulus. High stiffness and tensile strength of CNTs provide mechanical stability for electric nano-circuits formed by CNTs. The dielectric relaxation obeys ordinary alpha and beta relaxation of dielectrics at the current frequency range. High electrical conductivity may make (CNTs) highly recommended for many nano-electrical devices. Besides the mechanical properties in term of dimetral tensile and compression strength that are expressed by Young's modulus thatis closer to graphite. As a results we highly recommend these commercial (SWCTs) from our local suppliers for further medical applications.

Table 1. Mean values of calculated Young `s Moduli for CNTs of the current work for compression and diametrical tensile strength curves.

\begin{tabular}{llll}
\hline mean & Stress.max $\left(\mathbf{N} / \mathbf{m m}^{\mathbf{2}}\right)$ & strain \% & Young`s Modulus (GPa) \\
\hline Compression & 144.7315264 & 4.722222 & 3.064902911 \\
Dimetrial tensile & 9.033564815 & 2.105556 & 0.429034741 \\
\hline
\end{tabular}

\section{Acknowledgements}

Great appreciation is expressed for engineer Hakim Samuel (specialist in instrumental maintenance, faculty of dentistry, Alexandria University) for his valuable guidance in research procedure, and participation in data interpretation. The all thanks should be introduced to all staff of Ahmed Yameny 
laboratories especially, Dr. Ahmed Yameny, for his technical and laboratory assistance to make the work get the lights.

\section{References}

[1] Iijima, S (1991) Helical microtubules of graphitic carbon. Nature 354: $56-58$

[2] Anzar, N., Hasan, R., Tyagi, M., Yadav, N., \& Narang, J. (2020) Carbon nanotube - A review on Synthesis, Properties and plethora of applications in the field of biomedical science. Sensors International, 1: 100003.

[3] Banisaeid, M. (2020). Effect of functionalized carbon nanotubes on the mechanical properties of epoxy-based composites. Fullerenes, Nanotubes And Carbon Nanostructures, 28 (7), 582-588.

[4] Hasani, A. (2020). Approaches to Graphene, Carbon Nanotube and Carbon nanohorn, Synthesis, Properties and Applications. Nanoscience \& Nanotechnology-Asia, 10 (1): 4-11.

[5] Broza, G. (2010). Synthesis, properties, functionalisation and applications of carbon nanotube: a state of the art review. Chemistry \& Chemical Technology, 4 (1): 35-45.

[6] Eichhorn, W., \& Sezen, A. (2013). Flexible Carbon Nanotube Composite Sensors for Medical Device Application. Journal Of Medical Devices, 7 (2).

[7] Junkai L, Lianying A. (2020). Synthesis and Properties of Graphene/Carbon. Chemical Engineering Transactions, 71, 949-954.

[8] Kozinsky, B., \& Marzari, N. (2006). Static Dielectric Properties of Carbon Nanotubes from First Principles. Physical Review Letters, 96 (16).
[9] Kumar, A., Sharma, K., \& Dixit, A. (2019). Carbon nanotubeand graphene-reinforced multiphase polymeric composites: review on their properties and applications. Journal Of Materials Science, 55 (7), 2682-2724.

[10] Traves, C, Patigul, I, Fuyong, C, Gianlugi, A, Botton, I, and Alex, A. (2010). Synthesis and electrophoretic deposition of single-walled Carbon Nanotube Complexes with Conjugated Polyelectrolyte. Chem. Mater 22 (9), 2741-2749.

[11] Yu, Y., Cui, C., Qian, W., Xie, Q., Zheng, C., Kong, C., \& Wei, F. (2012). Carbon nanotube production and application in energy storage. Asia-Pacific Journal Of Chemical Engineering, 8 (2), 234-245.

[12] Ruoff, R., \& Lorents, D. (1995). Mechanical and thermal properties of carbon nanotubes. Carbon, 33 (7), 925-930.

[13] https://en.wikipedia.org/wiki/Buckypaper.acesssed 2020

[14] Salvetat-Delmotte, J., \& Rubio, A. (2002). Mechanical properties of carbon nanotubes: a fiber digest for beginners. Carbon, 40 (10), 1729-1734.

[15] Schwalke, U., \& Rispal, L. (2019). Fabrication of Ultra-Sensitive Carbon Nanotube Field-Effect Sensors (CNTFES) for Biomedical Applications. ECS Transactions, 13 (22), 39-45.

[16] Sheth, D., Maiti, S., Patel, S., Kandasamy, J., Chandan, M., \& Rahaman, A. (2020). Enhancement of mechanical properties of carbon fiber reinforced epoxy matrix laminated composites with multiwalled carbon nanotubes. Fullerenes, Nanotubes And Carbon Nanostructures, 1-7.

[17] Xiao, S., \& Hou, W. (2006). Studies of Size Effects on Carbon Nanotubes' Mechanical Properties by Using Different Potential Functions. Fullerenes, Nanotubes and Carbon Nanostructures, $14(1), 9-16$. 